\title{
Influence of the impurity scattering on charge transport in unconventional superconductor junctions
}

\author{
Bo Lu, ${ }^{1}$ Pablo Burset, ${ }^{1}$ Yasunari Tanuma, ${ }^{2}$ Alexander A. Golubov, ${ }^{3,6}$ Yasuhiro Asano, ${ }^{4,5,6}$ and Yukio Tanaka ${ }^{1,6}$ \\ ${ }^{1}$ Department of Applied Physics, Nagoya University, Nagoya 464-8603, Japan \\ ${ }^{2}$ Graduate School of Engineering Science, Akita University, Akita 010-8502, Japan \\ ${ }^{3}$ Faculty of Science and Technology and MESA+ Institute for Nanotechnology, University of Twente, 7500 AE Enschede, The Netherlands \\ ${ }^{4}$ Department of Applied Physics, Hokkaido University, Sapporo 060-8628, Japan \\ ${ }^{5}$ Center of Topological Science and Technology, Hokkaido University, Sapporo 060-8628, Japan \\ ${ }^{6}$ Moscow Institute of Physics and Technology, Dolgoprudny, Moscow 141700, Russia \\ (Received 23 April 2016; revised manuscript received 6 June 2016; published 5 July 2016)
}

\begin{abstract}
We study the influence of nonmagnetic impurity scatterings on the tunneling conductance of a junction consisting of a normal metal and a disordered unconventional superconductor by solving the quasiclassical Eilenberger equation self-consistently. We find that the impurity scatterings in both the Born and the unitary limits affect the formation of the Andreev bound states and modify strongly the tunneling spectra around zero bias. Our results are interpreted well by the appearance of odd-frequency Cooper pairs near the interface and by the divergent behavior of the impurity self-energy. The present paper provides a useful tool to identify the pairing symmetry of unconventional superconductors in experiments.
\end{abstract}

DOI: 10.1103/PhysRevB.94.014504

\section{INTRODUCTION}

The effects of impurity scatterings on superconducting phenomena are a key issue in the field of superconductivity. While the BCS $s$-wave pairing state is robust against nonmagnetic impurities [1], unconventional pairing states with other symmetries are usually rather fragile [2-7]. The impurity scatterings modify transport and thermodynamic properties of unconventional superconductors, which have a crucial impact on the identification of the pairing symmetry, e.g., in $p$-wave superconductors [8-10]. Specifically, the tunneling spectroscopy is an important experimental tool to identify an unconventional superconductor. The formation of the Andreev bound states at the surface of a superconductor is attributed to the anisotropy in the pairing states $[8,11-13]$ displaying a pronounced zero-bias conductance peak (ZBCP) in the tunneling conductance. In some cases, such bound states have a topological origin due to the bulk-edge correspondence [14-22]. Therefore, the ZBCP in the tunneling conductance strongly suggests unconventional pairing symmetries of a superconductor.

Theoretically, the tunneling spectroscopy is formulated as a differential conductance in a normal-metal-superconductor junction. Although effects of the potential disorder on the conductance have been studied in a number of papers, many of them focus on the disorder in the normal metal [23-26]. Surprisingly, only few attempts have been made to study the effects of disorder in the superconductor. Exceptional examples are the works on the proximity effect [27] and the ZBCP in high-Tc cuprate junctions, where it was found that the broadened ZBCP can be explained by the impurity scatterings in the superconductor or the surface roughness [28-31]. It is also interesting to investigate the disorder effect on the ZBCP for various pairing symmetries other than the $d$-wave case. For instance, it remains an open question whether the domelike subgap conductance in chiral $p$-wave superconductors [32-34] is robust in the presence of impurities. Similarly, chiral $d$-wave and chiral $f$-wave symmetries have been recently suggested to explain the absence of spontaneous edge currents in $\mathrm{Sr}_{2} \mathrm{RuO}_{4}$
[35,36], where the sensitivity of the chiral edge current to potential disorder depends on chiral pairing symmetries [37]. Several theoretical papers propose that doped graphene at a van Hove singular point may develop a chiral $d$-wave pairing state [38-41], which may be sensitive to weak impurity potentials treated within the Born limit. Furthermore, there is increasing evidence that suggests the strong impurity potential in $\mathrm{Sr}_{2} \mathrm{RuO}_{4}$ [42-45]. Theoretically, such strong impurity scatterings should be described by the unitary limit $[46,47]$. As a result, a plethora of novel systems require a detailed analysis of the impurity effects on unconventional pairings in both weak and strong impurity limits.

In this article, we present a self-consistent calculation of the tunneling spectra in a spin-degenerate normal-metaldisordered-superconductor junction with flat or chiral surface band states. For the former case, occurring in nodal superconductors, we find that the symmetry of the emergent odd-frequency pairing states $[26,48-51]$ plays a pivotal role in determining the evolution of the ZBCP under the impurity scatterings. As for chiral superconductors, our results show that the influence of the impurity scattering on the zero-bias conductance in the unitary limit is more pronounced than that in the Born limit. It is important to emphasize that the disorder strength we consider is still below the diffusive (or Usadel) limit [52]. In that case, the strong pair-breaking effect would make the unconventional superconductor gapless.

The remainder of this paper is organized as follows. In Sec. II, we describe our model and derive the essential formulas. In Sec. III, we discuss impurity effects in junctions with nodal superconductors. The order parameters and tunneling spectroscopy are studied. In Sec. IV, we show our result for chiral superconductors. Some concluding remarks are given in Sec. V.

\section{MODEL AND FORMALISM}

Let us consider a two-dimensional normal-metalsuperconductor junction with a flat interface at $x=0$ as shown in Fig. 1. The interface barrier potential is described 


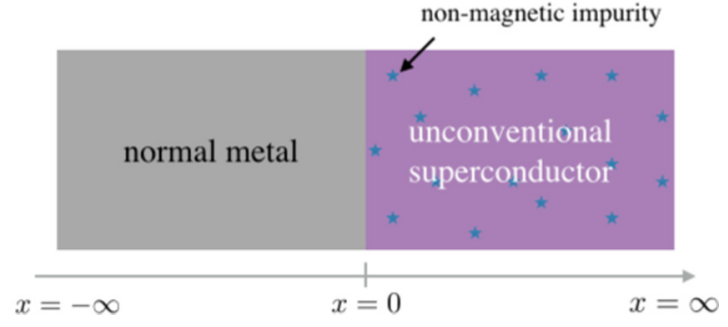

FIG. 1. Schematic illustration of a normal-metalunconventional-superconductor junction with a flat interface at $x=0$. We assume impurities in superconducting side.

by a $\delta$ function $V(x)=H \delta(x)$, with $H$ the potential strength. The normal metal $(x<0)$ is assumed to be clean, while the superconductor $(x>0)$ has a uniform impurity distribution. We denote the quasiclassical Green's function [53-56] by $\hat{g}^{\alpha \alpha}\left(i \omega_{n}, x, \theta_{\alpha}\right)$, with Matsubara frequency $\omega_{n}=(2 n+1) \pi T$, temperature $T$, and an integer $n$. For a given parallel momentum component, we define two angles $\theta_{ \pm}$corresponding to the Fermi momenta of the propagating quasiparticles. $\theta_{ \pm}$is given by $\theta_{+} \equiv \theta \in[-\pi / 2, \pi / 2]$ and $\theta_{-}=\pi-\theta$. We focus on the spin-degenerate system where $\hat{g}^{\alpha \alpha}\left(i \omega_{n}, x, \theta_{\alpha}\right)$ is a $2 \times 2$ matrix in particle-hole space satisfying Eilenberger equation [57]

$$
i v_{f} \cos \theta \partial_{x} \hat{g}^{\alpha \alpha}+\alpha\left[i \omega_{n} \hat{\tau}_{3}-\hat{\Delta}\left(\theta_{\alpha}, x\right) \hat{\tau}_{3}-\hat{a}(x), \hat{g}^{\alpha \alpha}\right]=0,
$$

accompanied by the normalization condition $\left(\hat{g}^{\alpha \alpha}\right)^{2}=-1$. Here $v_{f}$ is the quasiparticle Fermi velocity and $\hat{\tau}_{i}$ are Pauli matrices. $\hat{\Delta}\left(\theta_{\alpha}, x\right)$ and $\hat{a}(x)$ are the superconducting order parameter and the self-energy induced by impurities, respectively. In the normal-metal region, $\hat{\Delta}\left(\theta_{\alpha}, x<0\right)=\hat{a}(x<0)=$ 0 . We consider superconductors with different kinds of pairing symmetries. For $x>0$, it is convenient to define

$$
\hat{\Delta}\left(\theta_{\alpha}, x\right)=-\Delta_{1}\left(x, \theta_{\alpha}\right) \hat{\tau}_{2}+\Delta_{2}\left(x, \theta_{\alpha}\right) \hat{\tau}_{1} .
$$

For $d_{x y^{-}}, p_{x^{-}}$, and $p_{y^{-}}$-wave states, we choose the global U(1) gauge that makes $\hat{\Delta}\left(\theta_{\alpha}, x\right)$ a real matrix with $\Delta_{1}\left(x, \theta_{\alpha}\right) \equiv 0$. $\Delta_{2}\left(x, \theta_{\alpha}\right)$ is denoted by $\Delta(x) \chi\left(\theta_{\alpha}\right)$, where $\chi\left(\theta_{\alpha}\right)$ is chosen as $\cos \theta_{\alpha}$ ( $p_{x}$ wave $), \sin \theta_{\alpha}$ ( $p_{y}$ wave), and $\sin 2 \theta_{\alpha}\left(d_{x y}\right.$ wave). The self-consistency of $\Delta(x)$ is given in terms of the quasiclassical Green's function as

$$
\Delta(x)=\frac{2 T \sum_{0<\omega_{m}<\omega_{c}, \alpha} \int_{-\frac{\pi}{2}}^{\frac{\pi}{2}} d \theta^{\prime} \chi\left(\theta_{\alpha}^{\prime}\right)\left[\hat{g}^{\alpha \alpha}\left(i \omega_{m}, \theta_{\alpha}^{\prime}, x\right)\right]_{12}}{\ln \frac{T}{T_{c}}+\sum_{0<m<\frac{\omega_{c}}{2 \pi T}} \frac{1}{m-1 / 2}}
$$

where $T_{c}$ and $\omega_{c}$ are the critical temperature of the bulk superconductor and the Debye frequency, respectively. For chiral pairings, we choose a global U(1) gauge where the real component of $\hat{\Delta}\left(\theta_{\alpha}, x\right)$ is proportional to a cosine function of $\lambda \theta_{\alpha}$, with $\lambda=1,2,3$ corresponding to chiral $p$ waves, chiral $d$ waves, and chiral $f$ waves, respectively. We define $\Delta_{1}\left(x, \theta_{\alpha}\right)=$ $\Delta_{i m}(x) \chi_{i m}\left(\theta_{\alpha}\right)$ and $\Delta_{2}\left(x, \theta_{\alpha}\right)=\Delta_{r e}(x) \chi_{r e}\left(\theta_{\alpha}\right)$ with $\chi_{r e}\left(\theta_{\alpha}\right)=$ $\cos \left(\lambda \theta_{\alpha}\right)$ and $\chi_{i m}\left(\theta_{\alpha}\right)=\sin \left(\lambda \theta_{\alpha}\right) . \Delta_{r e / i m}(x)$ are determined by

$$
\Delta_{r e}(x)=\frac{\sum_{0<\omega_{m}<\omega_{c}, \alpha} \int_{-\frac{\pi}{2}}^{\frac{\pi}{2}} d \theta^{\prime} \chi_{r e}\left(\theta_{\alpha}^{\prime}\right)\left[\hat{g}^{\alpha \alpha}\left(i \omega_{m}, \theta_{\alpha}^{\prime}, x\right)\right]_{12}}{\frac{1}{2 T}\left(\ln \frac{T}{T_{c}}+\sum_{0<m<\frac{\omega_{c}}{2 \pi T}} \frac{1}{m-1 / 2}\right)}
$$

and

$$
\Delta_{i m}(x)=\frac{\sum_{0<\omega_{m}<\omega_{c}, \alpha} \int_{-\frac{\pi}{2}}^{\frac{\pi}{2}} d \theta^{\prime} \chi_{i m}\left(\theta_{\alpha}^{\prime}\right)\left[\hat{g}^{\alpha \alpha}\left(i \omega_{m}, \theta_{\alpha}^{\prime}, x\right)\right]_{12}}{\frac{i}{2 T}\left(\ln \frac{T}{T_{c}}+\sum_{0<m<\frac{\omega_{c}}{2 \pi T}} \frac{1}{m-1 / 2}\right)}
$$

The self-energy in the superconducting side can be decomposed as $\hat{a}=a_{1} \hat{\tau}_{1}+a_{2} \hat{\tau}_{2}+a_{3} \hat{\tau}_{3}$ and is connected to the quasiclassical Green's function by

$$
a_{j}\left(i \omega_{m}, x\right)=-\frac{1}{2 \tau} \frac{\frac{1}{1-\sigma} \sum_{\alpha}\left\langle g_{j}^{\alpha \alpha}\left(i \omega_{m}, x\right)\right\rangle_{\theta}}{1-\frac{\sigma}{1-\sigma} \sum_{i}\left[\sum_{\alpha}\left\langle g_{i}^{\alpha \alpha}\left(i \omega_{m}, x\right)\right\rangle_{\theta}\right]^{2}} .
$$

Here $1 / \tau$ and $\sigma$ denote the normal scattering rate and strength of a simple impurity potential, respectively. In the Born limit one finds that $\sigma \rightarrow 0$, while in the unitary limit $\sigma \rightarrow 1$, resulting in

$$
\hat{a}=\left\{\begin{array}{lr}
-\frac{1}{2 \tau} \sum_{\alpha}\left\langle\hat{g}^{\alpha \alpha}\right\rangle & \text { (Born limit) } \\
\frac{1}{2 \tau}\left[\sum_{\alpha}\left\langle\hat{g}^{\alpha \alpha}\right\rangle\right]^{-1} & \text { (unitary limit) }
\end{array}\right.
$$

using $\quad \hat{g}^{\alpha \alpha}=\sum_{i} g_{i}^{\alpha \alpha} \hat{\tau}_{i} \quad$ and $\quad \sum_{i}\left(\sum_{\alpha}\left\langle g_{i}^{\alpha \alpha}\right\rangle\right)^{2}=$ $\left[\sum_{i}\left(\sum_{\alpha}\left\langle g_{i}^{\alpha \alpha}\right\rangle\right) \hat{\tau}_{i}\right]^{2}$. In the superconducting region, the Riccati parametrization [58-60] is given by

$$
\begin{aligned}
\hat{g}^{++} & =\frac{i}{1-\mathcal{G}_{+}^{S} \mathcal{F}_{+}^{S}}\left[\begin{array}{cc}
1+\mathcal{G}_{+}^{S} \mathcal{F}_{+}^{S} & 2 i \mathcal{F}_{+}^{S} \\
2 i \mathcal{G}_{+}^{S} & -1-\mathcal{G}_{+}^{S} \mathcal{F}_{+}^{S}
\end{array}\right], \\
\hat{g}^{--} & =\frac{i}{\mathcal{G}_{-}^{S} \mathcal{F}_{-}^{S}-1}\left[\begin{array}{cc}
1+\mathcal{G}_{-}^{S} \mathcal{F}_{-}^{S} & 2 i \mathcal{F}_{-}^{S} \\
2 i \mathcal{G}_{-}^{S} & -1-\mathcal{G}_{-}^{S} \mathcal{F}_{-}^{S}
\end{array}\right] .
\end{aligned}
$$

The Riccati parameters $\mathcal{G}_{ \pm}^{S}, \mathcal{F}_{ \pm}^{S}$ obey the equations

$$
\begin{aligned}
& v_{f} \cos \theta \partial_{x} \mathcal{G}_{+}^{S}=2 \tilde{\omega}_{n} \mathcal{G}_{+}^{S}-\Lambda_{2+}+\Lambda_{1+}\left(\mathcal{G}_{+}^{S}\right)^{2}, \\
& v_{f} \cos \theta \partial_{x} \mathcal{F}_{+}^{S}=-2 \tilde{\omega}_{n} \mathcal{F}_{+}^{S}-\Lambda_{1+}+\Lambda_{2+}\left(\mathcal{F}_{+}^{S}\right)^{2} \\
& v_{f} \cos \theta \partial_{x} \mathcal{G}_{-}^{S}=-2 \tilde{\omega}_{n} \mathcal{G}_{-}^{S}+\Lambda_{2-}-\Lambda_{1-}\left(\mathcal{G}_{-}^{S}\right)^{2} \\
& v_{f} \cos \theta \partial_{x} \mathcal{F}_{-}^{S}=2 \tilde{\omega}_{n} \mathcal{F}_{-}^{S}+\Lambda_{1-}-\Lambda_{2-}\left(\mathcal{F}_{-}^{S}\right)^{2},
\end{aligned}
$$

with the definitions

$$
\begin{aligned}
\tilde{\omega}_{n} & =\omega_{n}+i a_{3} \\
\Lambda_{1 \pm} & =i \Delta_{1 \pm}+\Delta_{2 \pm}-a_{1}+i a_{2}, \\
\Lambda_{2 \pm} & =-i \Delta_{1 \pm}+\Delta_{2 \pm}+a_{1}+i a_{2} .
\end{aligned}
$$

At the interface $x=0$, we have the boundary conditions [58,60-62]

$$
\begin{aligned}
& \mathcal{F}_{+}^{S}(x=0) \rightarrow \begin{cases}\frac{R}{\mathcal{G}_{-}^{S}(x=0)}, & \omega_{n}>0, \\
\frac{1}{R \mathcal{G}_{-}^{S}(x=0)}, & \omega_{n}<0,\end{cases} \\
& \mathcal{F}_{-}^{S}(x=0) \rightarrow \begin{cases}\frac{1}{R \mathcal{G}_{+}^{S}(x=0)}, & \omega_{n}>0, \\
\frac{R}{\mathcal{G}_{+}^{S}(x=0)}, & \omega_{n}<0,\end{cases}
\end{aligned}
$$


with $R=Z^{2} /\left[Z^{2}+4 \cos ^{2} \theta\right], Z=2 m H / k_{f}, m$ the electron mass, and $k_{f}$ the Fermi momentum. In this work, we consider only the low transmitting case with fixed $Z=5$. The local density of states (LDOS) $\rho_{S}$ is given by

$$
\rho_{S}(x)=\frac{1}{\pi} \operatorname{Im} \sum_{\alpha} \int_{-\frac{\pi}{2}}^{\frac{\pi}{2}} d \theta^{\prime} \hat{g}^{\alpha \alpha}\left(i \omega_{m} \rightarrow E+i \delta, \theta_{\alpha}^{\prime}, x\right)_{11} .
$$

To obtain the conductance, we write down the wave functions in terms of the Riccati parameter $\overline{\mathcal{G}}_{ \pm}^{S}=\mathcal{G}_{ \pm}^{S}(E, \theta, x=0)$ at the interface, which are

$$
\left\{\begin{array}{l}
\mathrm{N}:\left[\begin{array}{l}
1 \\
a
\end{array}\right] e^{i k_{f} \cos \theta x}+\left[\begin{array}{l}
b \\
0
\end{array}\right] e^{-i k_{f} \cos \theta x}, \\
\mathrm{~S}: c\left[\begin{array}{c}
1 \\
i \overline{\mathcal{G}}_{+}^{S}
\end{array}\right] e^{i k_{f} \cos \theta x}+d\left[\begin{array}{c}
-i\left[\overline{\mathcal{G}}_{-}^{S}\right]^{-1} \\
1
\end{array}\right] e^{-i k_{f} \cos \theta x},
\end{array}\right.
$$

with $a, b(c, d)$ reflection (transmission) amplitudes. Using BTK theory [63], we obtain the normalized differential conductance

$$
\sigma_{t}=\int_{-\frac{\pi}{2}}^{\frac{\pi}{2}} d \theta \cos \theta \sigma_{\theta} / \sigma_{n},
$$

where $\sigma_{\theta}=1-|b|^{2}+|a|^{2}$ reads $[13,64]$

$$
\sigma_{\theta}=\sigma_{N} \frac{\left|\overline{\mathcal{G}}_{-}^{S}\right|^{2}+\sigma_{N}\left|\overline{\mathcal{G}}_{+}^{S} \overline{\mathcal{G}}_{-}^{S}\right|^{2}+\left(\sigma_{N}-1\right)\left|\overline{\mathcal{G}}_{+}^{S}\right|^{2}}{\left|\overline{\mathcal{G}}_{-}^{S}+\left(\sigma_{N}-1\right) \overline{\mathcal{G}}_{+}^{S}\right|^{2}},
$$

with $\sigma_{N}=4 \cos ^{2} \theta /\left[Z^{2}+4 \cos ^{2} \theta\right]$ and $\sigma_{n}=\int_{-\frac{\pi}{2}}^{\frac{\pi}{2}} d \theta \cos \theta \sigma_{N}$ the conductance in normal states. In the numerical calculations, we take the temperature $T=0.05 T_{c}$. For convenience, we do not consider thermodynamic phenomena and assume the Debye frequency $\omega_{c}=2 \pi T_{c}$ for all the considered pairing states.

\section{NODAL SUPERCONDUCTORS WITH FLAT SURFACE BANDS}

\section{A. Order parameter and odd frequency}

First, we show the spacial variation of the order parameter amplitude in the left panels of Fig. 2. Three regimes are considered: ballistic, Born, and unitary limit. In the ballistic case, the relaxation time becomes infinite so we set $1 / \tau=0$. For Born and unitary limits, it is given by $1 / \tau=0.2 \Delta_{b}$, where $\Delta_{b}=\Delta(\infty)$ is the bulk value of the order parameter (in ballistic case, $\Delta_{b}$ is denoted by $\Delta_{0}$ ). We define the superconducting coherence length as $\xi_{b}=v_{f} / \Delta_{b}$. We can see that for $p_{x}$ - and $d_{x y}$-wave cases, the order parameters with or without impurities strongly decrease when they approach the interface. However, for the $p_{y}$ case, the order parameter is almost invariant in space. For the following discussion of conductance spectra in this section, we plot the odd-frequency pairing states in the right panels of Fig. 2. The anomalous Green's function $\bar{F}=\sum_{\alpha}\left[\hat{g}^{\alpha \alpha}\right]_{12}$ can be decomposed into an even-frequency component $\bar{F}^{e}\left(i \omega_{n}, \theta\right)=\bar{F}^{e}\left(-i \omega_{n}, \theta\right)$ and an odd-frequency one $\bar{F}^{o}\left(i \omega_{n}, \theta\right)=-\bar{F}^{o}\left(-i \omega_{n}, \theta\right)$. The Fourier transform of $\bar{F}^{o(e)}$ is given by [65-67]

$$
\bar{F}^{o(e)}\left(i \omega_{n}, \theta\right)=\sum_{m} f_{c, m}^{o(e)} \cos (m \theta)+f_{s, m}^{o(e)} \sin (m \theta) .
$$
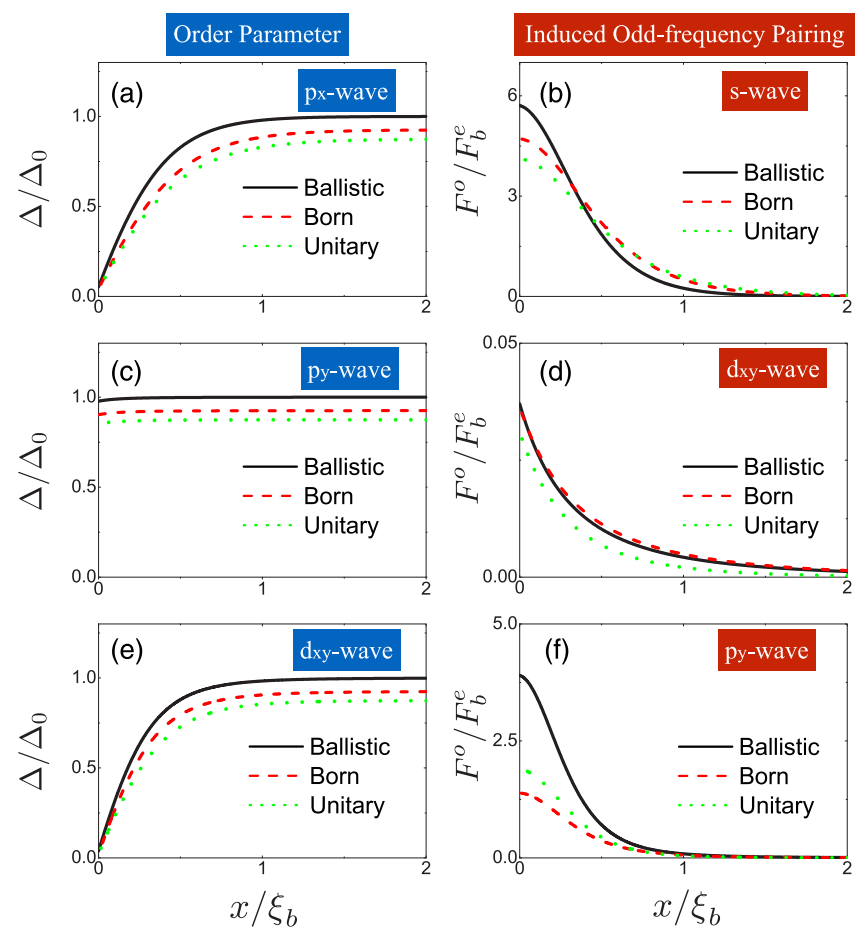

FIG. 2. Order parameter amplitude (left) and induced oddfrequency pairing amplitude (right) near the interface between a superconductor and a normal metal. From top to bottom: (a), (b) $p_{x}$ wave; (c), (d) $p_{y}$ wave; and (e), (f) $d_{x y}$ wave.

We denote by $F^{o}$ the dominant odd-frequency component $f_{c, m}^{o}$ or $f_{s, m}^{o}$ and normalize it by the bulk value of the even-frequency component $F_{b}^{e}=f_{c, 1}^{e}$ ( $p_{x}$ wave) or $F_{b}^{e}=f_{s, m}^{e}$ (with $m=1,2$ for $p_{y}, d_{x y}$ wave, respectively). It can be seen from the results that as the order parameter decreases in $p_{x}$ - and $d_{x y}$-wave cases, a large value of odd-frequency component is generated near the interface which determines the shape of Cooper pairs to be $s$ and $p_{y}$ wave, respectively [65]. One can compare the magnitudes of odd-frequency components in the presence of impurities with the ballistic case to find that the generated $s$-wave odd-frequency magnitude is not sensitive to the impurity scattering, which is consistent with the Anderson theorem [1]. In the $d_{x y}$-wave case, the non- $s$-wave odd-frequency component is strongly suppressed as the impurity scattering is introduced. For the $p_{y}$-wave case, the odd-frequency component is very small, while the dominant pairing near the surface is of even frequency.

\section{B. Tunneling spectroscopy}

We plot the normalized conductance $\sigma_{t}$ for a spin-triplet $p_{x}$ wave in Fig. 3. For comparison, we also show the non-selfconsistent (non-SCF) result (light gray line) and the LDOS $\rho_{t}=\rho_{S} / \rho_{n}$ at the interface $x=0$, with $\rho_{n}$ the density of states in the nonsuperconducting state. Although the $p_{x}$-wave superconductor has not been found in actual materials, it is becoming a topic of interest as a prototype for topological superconductivity [14-22] and an increasing effort is dedicated to designing $p_{x}$-wave superconductors using materials with strong spin-orbit coupling [68-73]. Our numerical calculation 

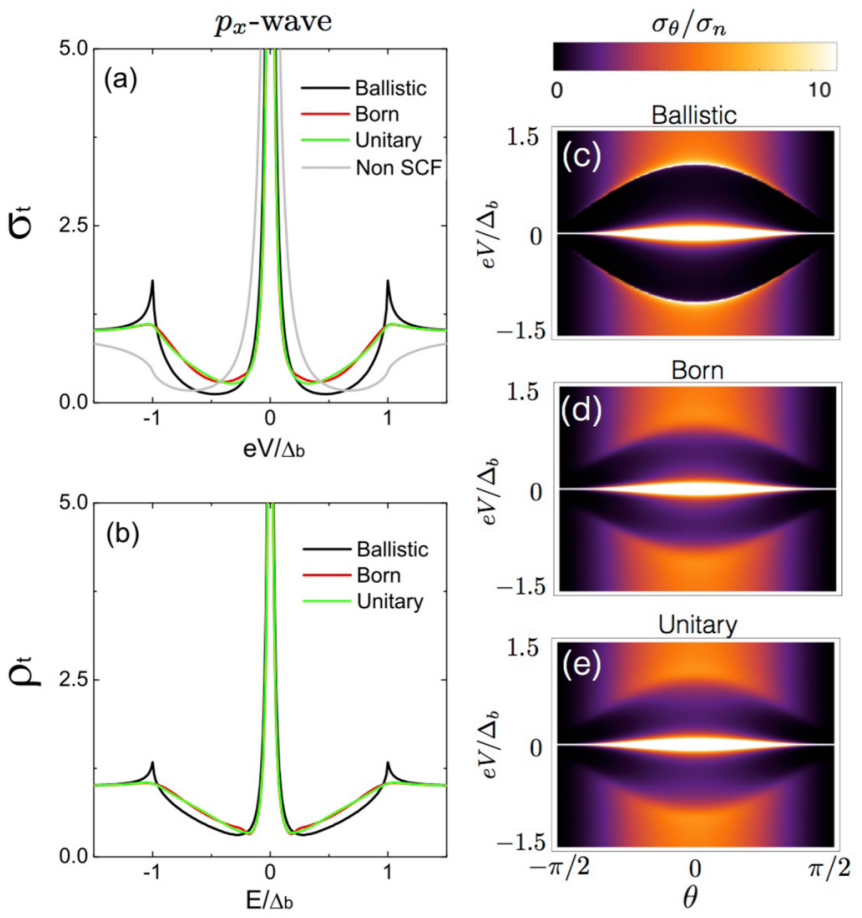

FIG. 3. $p_{x}$ wave: (a) conductance and (b) local density of states at the normal-metal-superconductor interface. Angle-resolved conductance for (c) ballistic, (d) Born, and (e) unitary limits.

of two-dimensional $p_{x}$-wave superconductors verifies the results of Ref. [73] about the robustness of the sharp ZBCP against impurities. Furthermore, we find that not only the height of ZBCP is robust but also the width does not broaden. For $e V \approx \Delta_{b}$, the ballistic case shows a sharp coherence peak which is smeared away in the presence of impurities. This is due to the energy level broadening by impurity effect, as concluded from the angle-resolved conductance spectra $\sigma_{\theta} / \sigma_{n}$ plotted in Figs. 3(d) and 3(e). For comparison, we reproduce the conductance result of Ref. [30] for $d_{x y}$-wave superconductors in Fig. 4. Although in the ballistic limit both $p_{x}$ - and $d_{x y}$-wave superconductors show a sharp ZBCP, their conductance spectra under impurity scattering are quite different. The ZBCP for $d_{x y}$ wave broadens and is suppressed by impurities, specially in the Born limit. Figures 4(d) and 4(e) show a smeared low-energy conductance in the angleresolved conductance spectra compared to the ballistic case of Fig. 4(c).

The impact of impurities on the ZBCP is shown in Fig. 5 for $p_{x}$ wave (black squares) and $d_{x y}$ wave (red triangles). Figures 5(a) and 5(b) show the evolution of the ZBCP as a function of the impurity scattering rate in the Born and unitary limits, respectively. The ZBCP for $p_{x}$-wave pairing is unaffected by changes in the scattering rate or the impurity strength, shown in Fig. 5(c). On the other hand, the ZBCP for $d_{x y}$ wave pairing is very vulnerable to the presence of impurities, becoming rapidly suppressed. Next, we show the results for $p_{y}$-wave junctions in Fig. 6. In this case, the Andreev bound state is absent, resulting in a sharp zero-bias conductance dip in the ballistic limit. For a fixed impurity
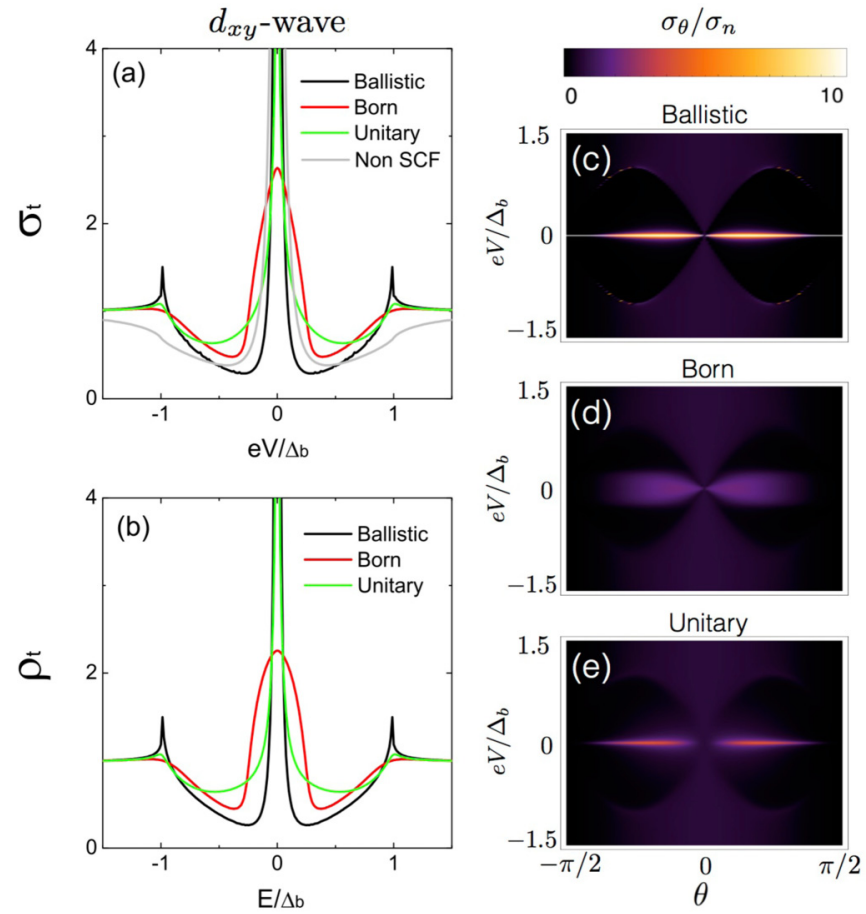

FIG. 4. $d_{x y}$ wave: (a) conductance and (b) local density of states at the normal-metal-superconductor interface. Angle-resolved conductance for (c) ballistic, (d) Born, and (e) unitary limits.

density level, we find that the dip structure is more pronounced in the Born limit while it is greatly smeared in the unitary limit.

These three typical behaviors of zero-bias conductance in nodal superconductors can be well interpreted from the
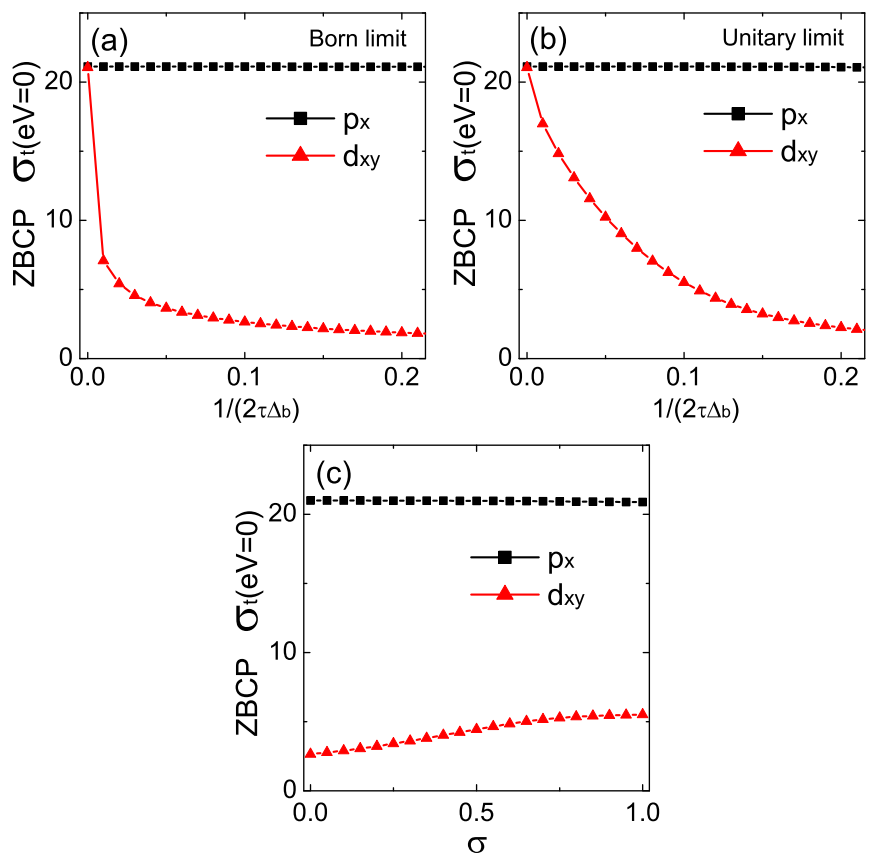

FIG. 5. Zero-bias conductance peak vs impurity scattering. (a) In the Born limit, the ZBCP as a function of the density of impurities and (b) the same plot in the unitary limit. (c) Fixing $1 /\left(2 \tau \Delta_{b}\right)$ at 0.1 , the $\mathrm{ZBCP}$ as a function of the impurity strength $\sigma$. 

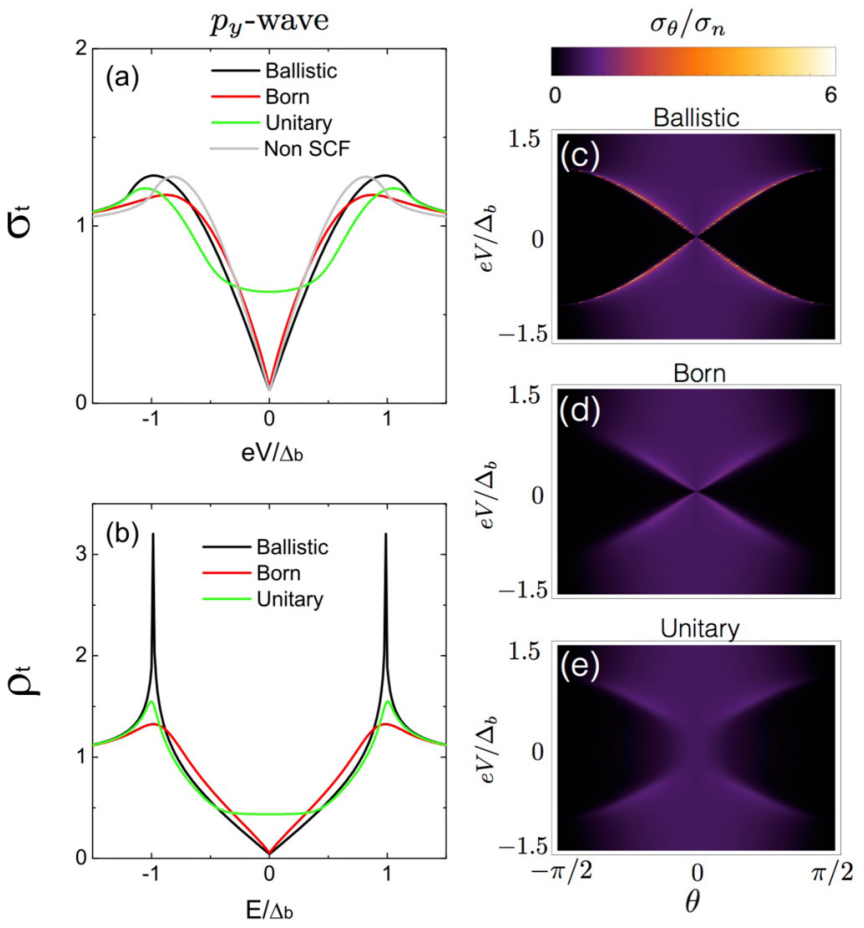

FIG. 6. $p_{y}$ wave: (a) conductance and (b) local density of states at the normal-metal-superconductor interface. Angle-resolved conductance for (c) ballistic, (d) Born, and (e) unitary limits.

symmetry of odd-frequency pairings and the divergent behavior of the self-energy. In the bulk, the Green's function $\hat{G}(E, \theta)=\sum_{\alpha} \hat{g}^{\alpha \alpha}\left(E, \theta_{\alpha}\right)$ is only divergent when the energy locates at continuum levels. However, in the junction system, the spacial dependent Green's function $\hat{G}(E, x, \theta)$ can also develop a divergence near the surface (or interface) $x=0$ due to the emergent Andreev bound state at $E=E_{b}, \theta=\theta_{b}$. For surface flat bands forming at angles connecting gap nodes, according to Eq. (4), the magnitude of the components $a_{i}$ of the self-energy $\hat{a}(E=0, x=0)$ in the Born limit is expected to surpass that of their counterparts in the unitary limit for the same scattering rate $1 / \tau$. Consequently, the ZBCP should be more sensitive to impurities in the Born limit than in the unitary limit, explaining the behavior of the $d_{x y}$-wave case. Further, we can also consider the symmetry of Cooper pairs near the interface. As previously discussed in Fig. 2, Cooper pairs at the interface of $p_{x}$-wave and $d_{x y}$-wave junctions are odd in frequency. For $p_{x}$ wave, Fermi-Dirac statistics impose that Cooper pairs form a spin-triplet isotropic $s$-wave state, making the pairings, together with the ZBCP, insensitive to impurities. This is a consequence of the ZBCP for $p_{x}$-wave junctions being protected by chiral symmetry [73]. For pairing symmetries like $d_{x y}$ wave, Cooper pairs form an odd-frequency spin-singlet $p$-wave pairing state which is dominant near the surface. Due to the anisotropy of the state, the $\mathrm{ZBCP}$ is more fragile. For pairing symmetries supporting no Andreev bound states, like the $p_{y}$-wave case, $\langle\hat{G}(E, x=0, \theta)\rangle_{\theta}$ is approximately zero when the energy $E$ is away from the continuum levels, which makes the self-energy $a_{i}$ stronger in the unitary limit than in the Born limit. Thus, the conductance is more sensitive in the unitary limit as shown in Fig. 6.

\section{GAPFUL SUPERCONDUCTORS WITH CHIRAL SURFACE BANDS}

In this section, we show the results of superconductors with chiral edge states. There is an increasing amount of evidence supporting that $\mathrm{Sr}_{2} \mathrm{RuO}_{4}$ is a candidate for a chiral $p$-wave superconductor [42-45]. The gap parameter has the form $\mathbf{d} \propto\left(k_{x}+i k_{y}\right)^{\lambda} \hat{\mathbf{e}}_{z}$, where $\hat{\mathbf{e}}_{z}$ is a unit vector along the tetragonal crystal $c$ axis and $\lambda=1$. However, such a chiral triplet superconductor features a spontaneous edge supercurrent which has not yet been observed in experiments. Several theories have suggested that $\lambda$ could be larger than 1, e.g., $\lambda=2$ (chiral $d$ wave) or $\lambda=3$ (chiral $f$ wave), leading to a suppression of the edge current $[35,36]$. Consequently, it is crucial to study the transport signatures of chiral $d$ - or chiral $f$-wave states in $\mathrm{Sr}_{2} \mathrm{RuO}_{4}$. The study of chiral $d$-wave superconducting states is also relevant since it has also been proposed in other systems, e.g., in doped graphene [38-41]. Additionally, designed chiral $p$-wave superconductivity has been suggested in semiconductor systems with spin-orbit couplings to realize topological states $[68,74]$.

First, we study the spacial dependence of the order parameters in chiral states. Since the order parameter is a complex number, it should be noted that we have chosen the global $\mathrm{U}(1)$ gauge, where $\operatorname{Re}\left[\hat{\Delta}\left(\theta_{\alpha}, x\right)\right]=\Delta_{r e}(x) \cos \left(\lambda \theta_{\alpha}\right) \hat{\tau}_{1}$. For the bulk state, we have $\Delta_{r e}(\infty)=\Delta_{i m}(\infty)=\Delta_{b}$, where $\Delta_{b}$ in the ballistic limit is denoted by $\Delta_{0}$ as in the previous section. Under this condition, we plot $\Delta_{r e}(x) / \Delta_{0}$ and $\Delta_{i m}(x) / \Delta_{0}$ in Fig. 7. It is found that chiral $p, d$, and $f$ waves display a similar behavior. The real and imaginary components of the order parameter vary in a different way as they approach the interface: One is slightly enhanced (real part for chiral $d$ wave and imaginary for chiral $p$ and $f$ wave), to a value denoted by $\Delta_{i n}$ at the interface, and the other is greatly suppressed. Such results have been revealed in previous studies of the BalianWerthamer (BW) phase of ${ }^{3} \mathrm{He}[8,75]$ or $\mathrm{Sr}_{2} \mathrm{RuO}_{4}$ [76-78].

We now proceed to discuss the conductance and LDOS. In Fig. 8, we show our results for chiral $p$-wave junctions. Compared to the non-SCF case (gray line), the dome-like ZBCP [32-34] is still visible with or without impurity scattering, although reduced. Actually, the height of the ZBCP has a very weak dependence on the impurity potential. A conductance peak appears near the bulk gap $e V \approx \Delta_{b}$, especially sharp in the ballistic limit. Also in the ballistic case, there are double peaks in the LDOS located at the position of $\Delta_{b}$ and $\Delta_{i n}$ [see Fig. 7(b)] [78]. The subgap LDOS at the interface is finite and almost constant, just slightly convex, revealing the existing chiral Andreev bound state across the region $-\Delta_{b}<E<\Delta_{b}$. We note that although the value of the $\mathrm{ZBCP}$ and the subgap conductance qualitatively agrees with the experiments, the obtained SCF result does not reproduce the full tunneling spectra in $\mathrm{Sr}_{2} \mathrm{RuO}_{4}[79,80]$. Thus, we consider that a self-consistent calculation in the framework of a three-band model [44] in $\mathrm{Sr}_{2} \mathrm{RuO}_{4}$ is needed in the future.

Figure 9 shows the LDOS and conductance spectroscopy for chiral $d$-wave superconductors. As can be seen from the LDOS in panel (b), the double peak structure outside the gap in the ballistic limit is prominent and still visible for the unitary case at $1 /\left(2 \tau \Delta_{b}\right)=0.1$, while the peak at $E=\Delta_{b}$ is absent in the Born limit. For the conductance spectroscopy 

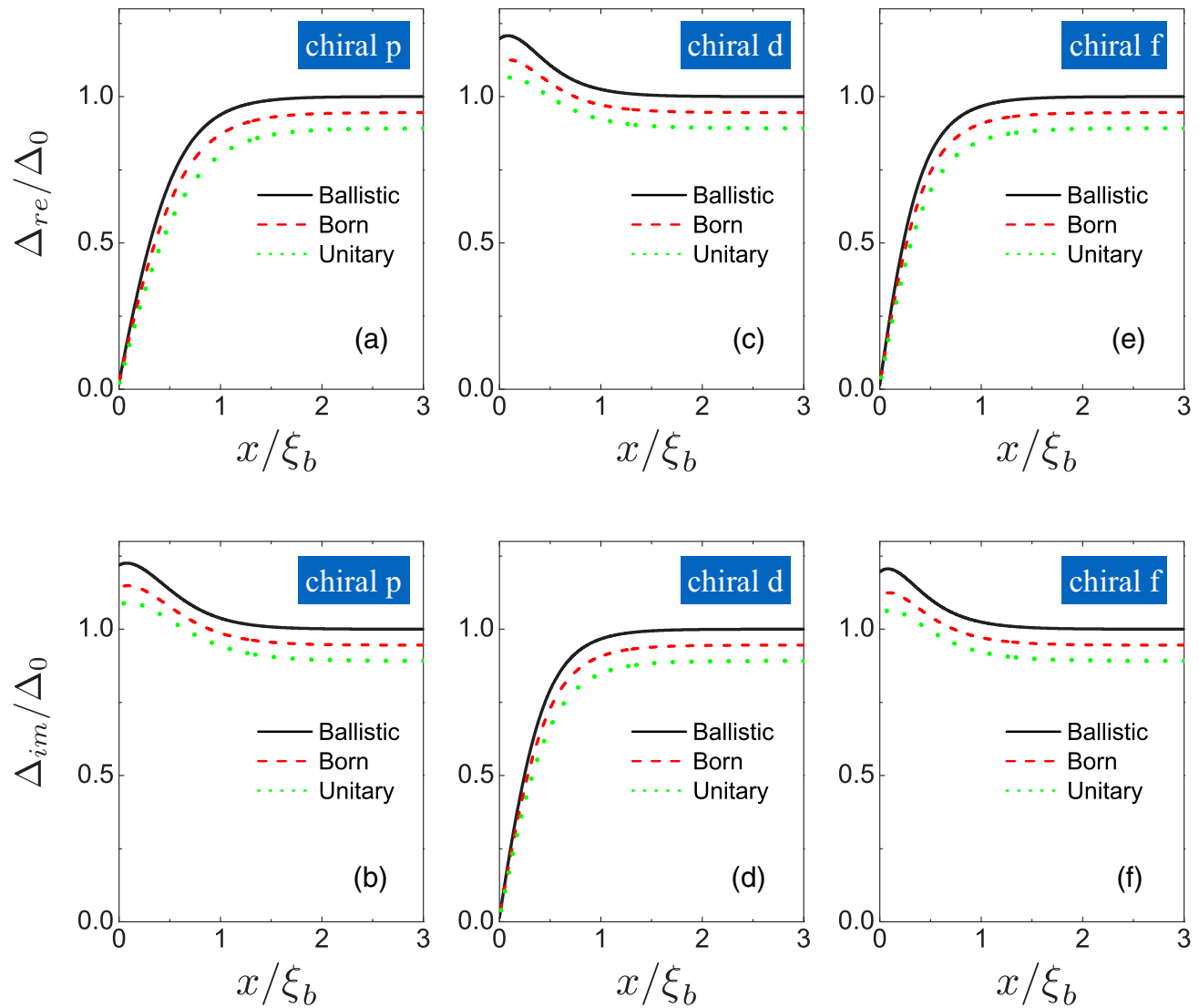

FIG. 7. Spacial dependence of order parameters in chiral wave superconductors: (a), (b) chiral $p$ wave; (c), (d) chiral $d$ wave; and (e), (f) chiral $f$ wave. The top panels show the real part of the order parameter and the bottom ones the imaginary part.
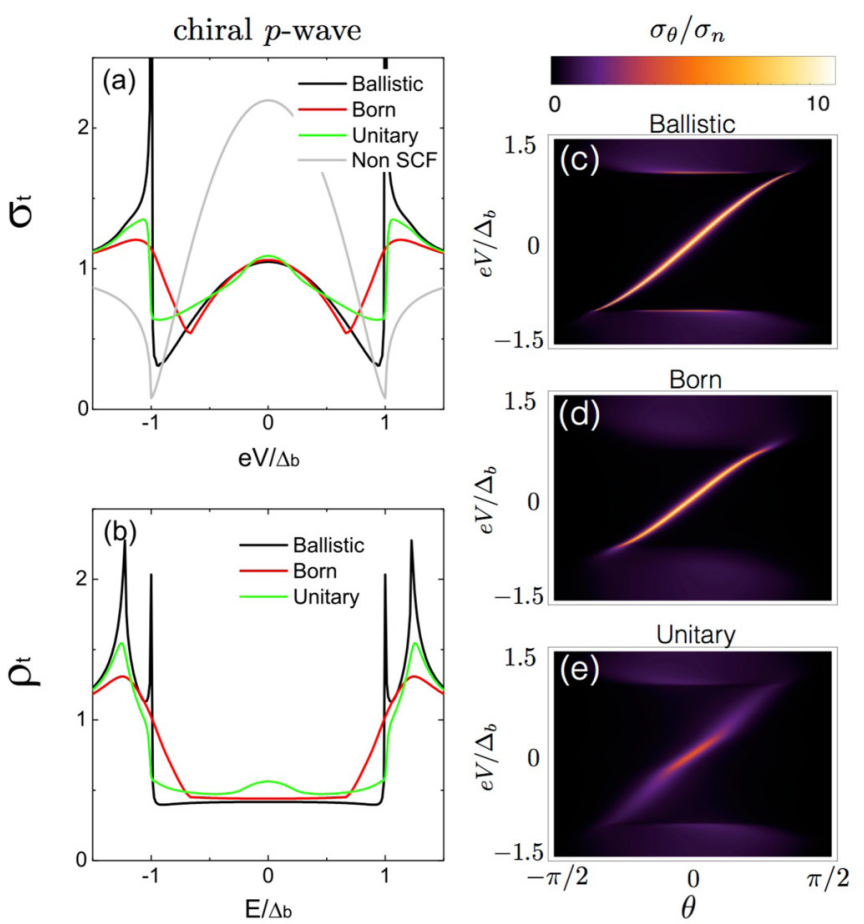

FIG. 8. Chiral $p$ wave: (a) conductance and (b) local density of states at the normal-metal-superconductor interface. Angle-resolved conductance for (c) ballistic, (d) Born, and (e) unitary limits.
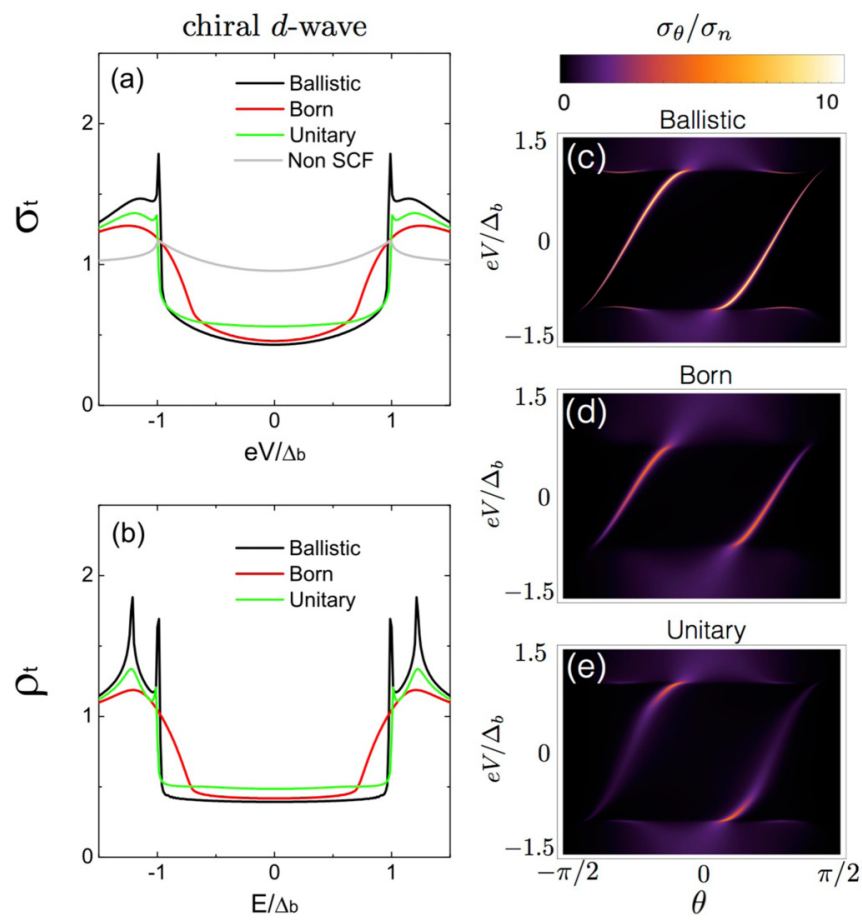

FIG. 9. Chiral $d$-wave: (a) conductance and (b) local density of states at the normal-metal-superconductor interface. Angle-resolved conductance for (c) ballistic, (d) Born, and (e) unitary limits. 

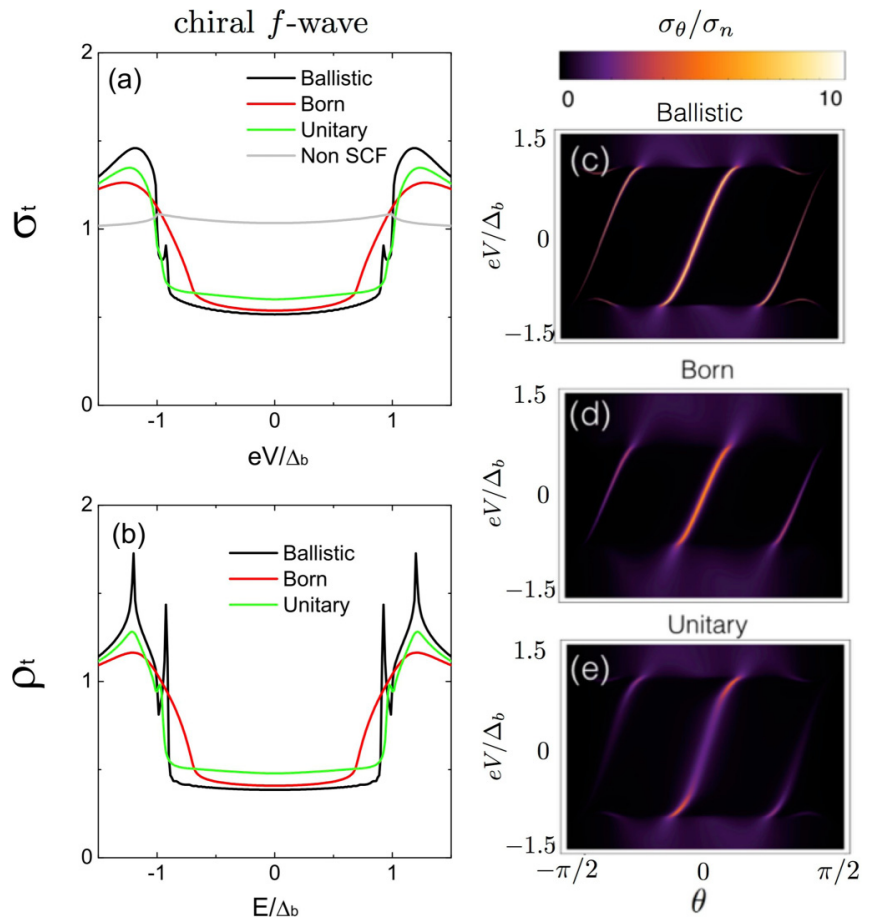

FIG. 10. Chiral $f$ wave: (a) conductance and (b) local density of states at the normal-metal-superconductor interface. Angle-resolved conductance for (c) ballistic, (d) Born, and (e) unitary limit.

shown in panel (a), we emphasize the absence of ZBCP. The conductance features a wide concave bottom gap and its minimum is barely affected by the impurity scattering. Besides that, the double-peak structure outside the gap is more visible in the conductance spectroscopy in the ballistic and unitary limits. The angle-resolved conductance spectra in Figs. 9(c)-9(e) display double-chiral states owing to a Chern number $c h=2$ [81,82].

Finally, we show the results for chiral $f$-wave states in Fig. 10. The subgap conductance and LDOS are similar to chiral $d$-wave states as seen in panels (a) and (b). In the ballistic limit, the conductance peak at $e V \approx \Delta_{b}$ becomes small as compared to chiral $p$-wave and chiral $d$-wave cases. The impurity effect does not change the bottomlike characteristic of conductance and LDOS inside the bulk gap. Here the Chern number $c h=3$ manifests as three chiral Andreev bound states inside the gap as seen in Figs. 10(c)-10(e). However, the slopes of the states are much higher than those of chiral $p$ and chiral $d$-wave pairings, reducing their contribution to the angle-averaged conductance. As a result, there are no ZBCP for the parameters we have used.

Due to the characteristics of the tunneling spectroscopy and LDOS for chiral superconductors, it is convenient to discuss the impurity effect in three separate energy regions: (1) $E \sim 0$, (2) $E \sim \Delta_{b}$, (3) $E \sim \Delta_{i n}$. Similarly to the previous section, let us focus on the divergence of the Green's function $\hat{G}(E, \theta)$. Compared to the flat surface bands, the density of zero-energy states is greatly reduced, leading to a finite but relatively small value of angle-averaged Green's function $\langle\hat{G}(E=0, x=0, \theta)\rangle_{\theta}$. Thus, for the same scattering rate $1 / \tau$, the magnitude of the components $a_{i}$ of the self-energy
TABLE I. Zero-bias conductance vs impurity scattering in the ballistic, Born, and unitary limits. The label $\bigcirc$ indicates the immunity to impurity scattering. $\simeq(\sim)$ means the zero-bias conductance is approximately similar (slightly increased). - (--) means a decrease (strong decrease) of conductance with respect to the ballistic limit.

\begin{tabular}{cccc}
\hline \hline & Ballistic $\left[\sigma_{t}(e V=0)\right]$ & Born & Unitary \\
\hline$p_{x}$ & Peak $\left(\sigma_{t} \gg 1\right)$ & $\bigcirc$ & $\bigcirc$ \\
$p_{y}$ & $\operatorname{Dip}\left(\sigma_{t}=0\right)$ & $\simeq$ & $\sim$ \\
$d_{x y}$ & $\operatorname{Peak}\left(\sigma_{t} \gg 1\right)$ & -- & - \\
Chiral $p$ & Peak $\left(\sigma_{t} \sim 1\right)$ & $\simeq$ & $\sim$ \\
Chiral $d$ & Enhancement $\left(0<\sigma_{t}<1\right)$ & $\simeq$ & $\sim$ \\
Chiral $f$ & Enhancement $\left(0<\sigma_{t}<1\right)$ & $\simeq$ & $\sim$ \\
\hline \hline
\end{tabular}

$\hat{a}(E=0, x=0)$ is more pronounced in the unitary limit than in the Born limit. Consequently, the zero-bias conductance and LDOS are more sensitive in the unitary limit. For $E \sim \Delta_{b}$ and $E \sim \Delta_{i n}$, the appearance of continuum bands greatly enhances $\langle\hat{G}(E, x=0, \theta)\rangle_{\theta}$ and makes the self-energy terms $a_{i}$ smaller. As a result, the unitary limit would retain the double peak structure mostly outside the bulk gap $\Delta_{b}$ [e.g., see panel (b) of Fig. 9]. It is also easy to understand that the steep structure in the conductance and LDOS near $E \sim \Delta_{b}$ in the ballistic limit disappears easily in the Born limit. We have summarized the behavior of the zero-bias conductance in Table I.

\section{CONCLUSION}

In conclusion, we have theoretically studied the tunneling spectroscopy in normal-metal-disordered-unconventionalsuperconductor junctions at low transparency for various pairing symmetries. For nodal superconductors with flat surface bands, the influence of the impurity scattering can be classified into three types according to the behavior of zerobias conductance: (1) When Cooper pairs at the surface have odd-frequency $s$-wave symmetry, the zero-bias conductance peak is immune to the impurity scattering; (2) for non- $s$-wave odd-frequency pairing, the zero-bias conductance peak is strongly suppressed by impurities; and (3) in the accidental absence of Andreev bound states, the zero-bias conductance dip is easily smeared by impurities in the unitary limit.

For chiral superconductors, we have shown the selfconsistent result of the conductance spectra and found that a zero-bias conductance peak for chiral $p$-wave pairings remains in the presence of impurities. The zero-bias conductance for chiral waves is more sensitive to impurity scattering in the unitary limit than in the Born limit. Moreover, the double peaks occurring in the LDOS and conductance spectra at the gap edges for all chiral waves are very sensitive to impurity scattering and are especially blurred in the Born limit. We expect that our results can be exploited for the experimental identification of pairing symmetries by analyzing the tunneling spectroscopy of unconventional superconductors.

Finally, even though we only discuss the two-dimensional spin-degenerate superconductors, some extension can be made to study one- or three-dimensional cases by analyzing the symmetry of induced pair amplitude and the poles of selfenergies. Furthermore, in the light of the recent developments of Eilenberger theory including spin-orbit coupling (see, e.g., 
Refs. [83-87]), an interesting subject for future study is spinorbit coupling in hybrid systems with impurities.

\section{ACKNOWLEDGMENTS}

We thank S. V. Bakurskiy, M. Y. Kupriyanov, and K. Yada for valuable discussions. This work was supported by a Grant-in-Aid for Scientific Research on Innovative Areas Topological Material Science (Grants No. 15H05851, No. 15H05852, and No. 15H05853), a Grant-in-Aid for
Scientific Research B (Grant No. 15H03686), a Grant-in-Aid for Challenging Exploratory Research (Grant No. 15K13498) from the Ministry of Education, Culture, Sports, Science, and Technology, Japan (MEXT); Japan-RFBR JSPS Bilateral Joint Research Projects/Seminars (Grants No. 15-52-50054 and No. 15668956); and Dutch FOM, the Ministry of Education and Science of the Russian Federation, Grant No. 14.Y26.31.0007, and by the Russian Science Foundation, Grant No. 1512-30030. P.B. acknowledges support from Japan Society for the Promotion of Science (JSPS) International Research Fellowship.
[1] P. W. Anderson, J. Phys. Chem. Solids 11, 26 (1959).

[2] A. I. Larkin, Zh. Eksp. Teor. Fiz. 2, 205 (1965) [JETP Lett. 2, 130 (1965)].

[3] R. Balian and N. R. Werthamer, Phys. Rev. 131, 1553 (1963).

[4] A. J. Millis, S. Sachdev, and C. M. Varma, Phys. Rev. B 37, 4975 (1988).

[5] M. Sigrist and K. Ueda, Rev. Mod. Phys. 63, 239 (1991).

[6] R. J. Radtke, K. Levin, H. B. Schuttler, and M. R. Norman, Phys. Rev. B 48, 653 (1993).

[7] A. V. Balatsky, I. Vekhter, and J.-X. Zhu, Rev. Mod. Phys. 78, 373 (2006).

[8] L. J. Buchholtz and G. Zwicknagl, Phys. Rev. B 23, 5788 (1981).

[9] K. Maki and E. Puchkaryov, Europhys. Lett. 50, 533 (2000).

[10] M. J. Graf and A. V. Balatsky, Phys. Rev. B 62, 9697 (2000).

[11] C. Bruder, Phys. Rev. B 41, 4017 (1990).

[12] C. R. Hu, Phys. Rev. Lett. 72, 1526 (1994).

[13] S. Kashiwaya and Y. Tanaka, Rep. Prog. Phys. 63, 1641 (2000).

[14] A. P. Schnyder, S. Ryu, A. Furusaki, and A. W. W. Ludwig, Phys. Rev. B 78, 195125 (2008).

[15] M. Sato, Phys. Rev. B 79, 214526 (2009).

[16] M. Sato, Phys. Rev. B 81, 220504(R) (2010).

[17] M. Z. Hasan and C. L. Kane, Rev. Mod. Phys. 82, 3045 (2010).

[18] S. Ryu, A. P. Schnyder, A. Furusaki, and A. W. W. Ludwig, New J. Phys. 12, 065010 (2010).

[19] M. Z. Hasan and J. E. Moore, Annu. Rev. Condens. Matter Phys. 2, 55 (2011).

[20] X. L. Qi and S. C. Zhang, Rev. Mod. Phys. 83, 1057 (2011).

[21] J. Alicea, Rep. Prog. Phys. 75, 076501 (2012).

[22] Y. Tanaka, M. Sato, and N. Nagaosa, J. Phys. Soc. Jpn. 81, 011013 (2012).

[23] Y. Tanaka, Y. V. Nazarov, and S. Kashiwaya, Phys. Rev. Lett. 90, 167003 (2003).

[24] Y. Tanaka and S. Kashiwaya, Phys. Rev. B 70, 012507 (2004).

[25] Y. Tanaka, S. Kashiwaya, and T. Yokoyama, Phys. Rev. B 71, 094513 (2005).

[26] Y. Tanaka and A. A. Golubov, Phys. Rev. Lett. 98, 037003 (2007).

[27] T. Löfwander, Phys. Rev. B 70, 094518 (2004).

[28] A. Poenicke, Y. S. Barash, C. Bruder, and V. Istyukov, Phys. Rev. B 59, 7102 (1999).

[29] A. A. Golubov and M. Yu. Kupriyanov, Pis'ma Zh. Eksp. Teor. Fiz. 69, 242 (1999) [JETP Lett. 69, 262 (1999)].

[30] Y. Tanaka, Y. Tanuma, and S. Kashiwaya, Phys. Rev. B 64, 054510 (2001).

[31] Y. Asano and Y. Tanaka, Phys. Rev. B 65, 064522 (2002).
[32] M. Yamashiro, Y. Tanaka, and S. Kashiwaya, Phys. Rev. B 56, 7847 (1997).

[33] C. Honerkamp and M. Sigristt, J. Low Temp. Phys. 111, 895 (1998).

[34] M. Yamashiro, Y. Tanaka, Y. Tanuma, and S. Kashiwaya, J. Phys. Soc. Jpn. 67, 3224 (1998).

[35] W. Huang, E. Taylor, and C. Kallin, Phys. Rev. B 90, 224519 (2014).

[36] Y. Tada, W. Nie, and M. Oshikawa, Phys. Rev. Lett. 114, 195301 (2015).

[37] S.-I. Suzuki and Y. Asano, arXiv:1602.03994.

[38] A. M. Black-Schaffer and C. Honerkamp, J. Phys.: Condens. Matter 26, 423201 (2014).

[39] R. Nandkishore, L. S. Levitov, and A. V. Chubukov, Nat. Phys. 8, 158 (2012).

[40] W. S. Wang, Y. Y. Xiang, Q. H. Wang, F. Wang, F. Yang, and D. H. Lee, Phys. Rev. B 85, 035414 (2012).

[41] M. L. Kiesel, C. Platt, W. Hanke, D. A. Abanin, and R. Thomale, Phys. Rev. B 86, 020507(R) (2012).

[42] Y. Maeno, H. Hashimoto, K. Yoshida, S. Nishizaki, T. Fujita, J. G. Bednorz, and F. Lichtenberg, Nature (London) 372, 532 (1994).

[43] C. Kallin, Rep. Prog. Phys. 75, 042501 (2012).

[44] A. P. Mackenzie and Y. Maeno, Rev. Mod. Phys. 75, 657 (2003).

[45] Y. Maeno, S. Kittaka, T. Nomura, S. Yonezawa, and K. Ishida, J. Phys. Soc. Jpn. 81, 011009 (2012).

[46] K. Miyake and O. Narikiyo, Phys. Rev. Lett. 83, 1423 (1999).

[47] M. Suzuki, M. A. Tanatar, N. Kikugawa, Z. Q. Mao, Y. Maeno, and T. Ishiguro, Phys. Rev. Lett. 88, 227004 (2002).

[48] V. L. Berezinskii, Pis'ma Zh. Eksp. Teor. Fiz. 20, 628 (1974) [JETP Lett. 20, 287 (1974)].

[49] T. R. Kirkpatrick and D. Belitz, Phys. Rev. Lett. 66, 1533 (1991).

[50] A. Balatsky and E. Abrahams, Phys. Rev. B 45, 13125 (1992).

[51] F. S. Bergeret, A. F. Volkov, and K. B. Efetov, Rev. Mod. Phys. 77, 1321 (2005).

[52] K. D. Usadel, Phys. Rev. Lett. 25, 507 (1970).

[53] J. W. Serene and D. Rainer, Phys. Rep. 101, 221 (1983).

[54] A. V. Zaitsev, Zh. Eksp. Teor. Fiz. 86, 1742 (1984) [Sov. Phys. JETP 59, 1015 (1984)].

[55] A. Millis, D. Rainer, and J. A. Sauls, Phys. Rev. B 38, 4504 (1988).

[56] M. Ashida, S. Aoyama, J. Hara, and K. Nagai, Phys. Rev. B 40, 8673 (1989).

[57] G. Eilenberger, Z. Phys. 214, 195 (1968). 
[58] Y. Nagato, K. Nagai, and J. Hara, J. Low Temp. Phys. 93, 33 (1993).

[59] N. Schopohl and K. Maki, Phys. Rev. B 52, 490 (1995).

[60] M. Eschrig, Phys. Rev. B 61, 9061 (2000).

[61] M. Fogelström, Phys. Rev. B 62, 11812 (2000).

[62] E. Zhao, T. Löfwander, and J. A. Sauls, Phys. Rev. B 70, 134510 (2004).

[63] G. E. Blonder, M. Tinkham, and T. M. Klapwijk, Phys. Rev. B 25, 4515 (1982).

[64] Y. Tanaka and S. Kashiwaya, Phys. Rev. Lett. 74, 3451 (1995).

[65] Y. Tanaka, A. A. Golubov, S. Kashiwaya, and M. Ueda, Phys. Rev. Lett. 99, 037005 (2007).

[66] M. Eschrig, T. Löfwander, T. Champel, J. C. Cuevas, J. Kopu, and G. Schön, J. Low Temp. Phys. 147, 457 (2007).

[67] Y. Tanaka, Y. Tanuma, and A. A. Golubov, Phys. Rev. B 76, 054522 (2007).

[68] J. D. Sau, R. M. Lutchyn, S. Tewari, and S. Das Sarma, Phys. Rev. Lett. 104, 040502 (2010).

[69] R. M. Lutchyn, J. D. Sau, and S. Das Sarma, Phys. Rev. Lett. 105, 077001 (2010).

[70] Y. Oreg, G. Refael, and F. von Oppen, Phys. Rev. Lett. 105, 177002 (2010).

[71] J. You, C. H. Oh, and V. Vedral, Phys. Rev. B 87, 054501 (2013).

[72] H.-Y. Hui, J. D. Sau, and S. Das Sarma, Phys. Rev. B 90, 064516 (2014).

[73] S. Ikegaya, Y. Asano, and Y. Tanaka, Phys. Rev. B 91, 174511 (2015).
[74] J. Alicea, Phys. Rev. B 81, 125318 (2010).

[75] S. Higashitani, Y. Nagato, and K. Nagai, J. Low Tem. Phys. 155, 83 (2009).

[76] M. Matsumoto and M. Sigrist, J. Phys. Soc. Jpn. 68, 994 (1999).

[77] Y. Tanuma, Y. Tanaka, and S. Kashiwaya, Phys. Rev. B 74, 024506 (2006).

[78] S. V. Bakurskiy, A. A. Golubov, M. Y. Kupriyanov, K. Yada, and Y. Tanaka, Phys. Rev. B 90, 064513 (2014).

[79] F. Laube, G. Goll, H. v. Löhneysen, M. Fogelström, and F. Lichtenberg, Phys. Rev. Lett. 84, 1595 (2000).

[80] S. Kashiwaya, H. Kashiwaya, H. Kambara, T. Furuta, H. Yaguchi, Y. Tanaka, and Y. Maeno, Phys. Rev. Lett. 107, 077003 (2011).

[81] G. E. Volovik, JETP Lett. 70, 609 (1999).

[82] S. Kashiwaya, H. Kashiwaya, K. Saitoh, Y. Mawatari, and Y. Tanaka, Phys. E (Amsterdam, Neth.) 55, 25 (2014).

[83] N. Hayashi, K. Wakabayashi, P. A. Frigeri, and M. Sigrist, Phys. Rev. B 73, 024504 (2006).

[84] R. Raimondi, C. Gorini, P. Schwab, and M. Dzierzawa, Phys. Rev. B 74, 035340 (2006).

[85] A. B. Vorontsov, I. Vekhter, and M. Eschrig, Phys. Rev. Lett. 101, 127003 (2008).

[86] F. S. Bergeret and I. V. Tokatly, Phys. Rev. Lett. 110, 117003 (2013).

[87] F. Konschelle, I. V. Tokatly, and F. S. Bergeret, Phys. Rev. B 92, 125443 (2015) 